\title{
Low expression of the $X$-linked ribosomal protein S4 in human serous epithelial ovarian cancer is associated with a poor prognosis
}

Serges P Tsofack', Liliane Meunier, ${ }^{2,3}$, Lilia Sanchez ${ }^{3}$, Jason Madore, ${ }^{2,3}$, Diane Provencher ${ }^{2,3}$, Anne-Marie Mes-Masson ${ }^{2,3}$ and Michel Lebel ${ }^{1,4^{*}}$

\begin{abstract}
Background: The X-linked ribosomal protein S4 (RPS4X), which is involved in cellular translation and proliferation, has previously been identified as a partner of the overexpressed multifunctional protein YB-1 in several breast cancer cells. Depletion of RPS4X results in consistent resistance to cisplatin in such cell lines.

Methods: As platinum-based chemotherapy is a standard first line therapy used to treat patients with ovarian cancer, we evaluated the prognostic value of RPS4X and YB-1 at the protein level in specimen from 192 high-grade serous epithelial ovarian cancer patients.

Results: Immunohistochemistry studies indicated that high expression of RPS4X was associated with a lower risk of death and later disease progression ( $H R=0.713, P=0.001$ and $H R=0.761, P=0.001$, respectively) as compared to low expression of RPS4X. In contrast, YB-1 was not significantly associated with either recurrence or survival time in this cohort. Finally, the depletion of RPS4X with different siRNAs in two different ovarian cancer cell lines reduced their proliferative growth rate but more importantly increased their resistance to cisplatin.

Conclusion: Altogether, these results suggest that the levels of RPS4X could be a good indicator for resistance to platinum-based therapy and a prognostic marker for ovarian cancer. Our study also showed that RPS4X is an independent prognostic factor in patients with serous epithelial ovarian cancer.
\end{abstract}

Keywords: Serous epithelial ovarian cancer, YB-1, RPS4X, Cisplatin

\section{Background}

Epithelial ovarian cancer (EOC) is a disease responsible for more cancer deaths among women in the Western world than all other gynecologic malignancies. Because of its asymptomatic nature, ovarian cancer is characterized at presentation with advanced disease having spread primarily via an intraperitoneal route. An initial surgical approach is essential for proper staging of the disease process and for aggressive cytoreduction, which in turn improves the response to chemotherapy and survival [1]. Chemotherapy has had an increasingly important role in

\footnotetext{
* Correspondence: michel.lebel@crhdq.ulaval.ca

${ }^{1}$ Centre de Recherche en Cancérologie de I'Université Laval, Hôpital

Hôtel-Dieu de Québec, Quebec City, QC, Canada

${ }^{4}$ Centre de Recherche en Cancérologie de I'Université Laval, Hôpital

Hôtel-Dieu de Québec, 9 McMahon Sreet, Quebec City, QC G1R 2J6, Canada Full list of author information is available at the end of the article
}

the effective treatment of ovarian cancer. The reference standard for postsurgical ovarian cancer first-line chemotherapy has been the use of a platinum-taxane combination [2]. Although the standard platinumtaxane regimen results in a response rate of $80 \%$ in advanced ovarian cancer patients, most of these patients relapse after a median period of 18 months, due to the emergence of tumor resistance to these conventional drugs [3-5]. Thus, there is an immediate need for the identification of pharmacogenomic markers to identify patients unlikely to respond, those that will relapse rapidly, or patients at risk for severe toxicity.

In recent years, several studies have reported the involvement of YB-1 in patient survival and cisplatin resistance in ovarian cancers $[6,7]$. The YB-1 protein is a multifunctional protein that affects the transcription, splicing, and translation of specific mRNAs [8-11]. Increased expression of

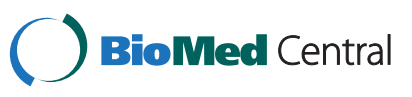


YB-1 is associated with a poor prognosis in ovarian cancer [7]. YB-1 binds preferentially to cisplatin-modified DNA [12] and interacts with several DNA repair proteins $[13,14]$. Although YB-1 affects several biological processes, it is still unknown which ones are important for cisplatin resistance. In a recent study of breast cancer cell lines, we identified the proteins that interact directly to YB-1 and impact on cisplatin response upon depletion [15]. Interestingly, we found that the small ribosomal protein $4 \mathrm{X}$ (RPS4X) increases cisplatin resistance upon depletion with specific small interference RNAs. As platinum-based compounds are used in the treatment of ovarian cancers, we sought to correlate the levels of RPS4X in clinical samples with patient survival and disease progression.

In this work, we determined by immunohistochemistry the levels of both RPS4X and YB-1 in ovarian cancer samples from patients who were treated with a platinum-based chemotherapeutic regimen after their surgery. RPS4X not only correlated with stage, but low levels of RPS4X also correlated with poor survival and disease progression. These results indicate that RPS4X could be a predictive and prognostic marker in ovarian cancer.

\section{Methods}

\section{Ethics statement}

Ethics approval for specimen collection and the study were obtained by the local institutional ethics board (Comité d'éthique de la recherche du Centre hospitalier de l’Université de Montréal).

\section{Patients and tissue specimens}

Tumor samples were collected and banked following appropriate consent from patients undergoing surgery within the Division of Gynecologic Oncology at the Centre hospitalier de l'Université de Montréal from 1993 to 2010. An independent dedicated GYN-pathologist scored the tumor grade and subtype and a gynecologic oncologist scored the stage and the tumor residual disease according to criteria from the International Federation of Gynecologists and Obstetricians [16]. Clinical data on progressionfree interval were defined according to RECIST 1.1 [17]. Overall survival was defined as the time from surgery to death from ovarian cancer. Patients known to be still alive at time of analysis were censored at time of their last follow-up. Patient disease free survival (DFS) was calculated from the time of surgery until the first progression. Eligibility criteria for inclusion in the study were as follows: primary surgery, complete information on post-operative chemotherapeutic treatment, high grade serous histopathology subtype, and completed tumor banking informed consent. Patients who died from another disease were censored at time of last follow-up. A gynecologic oncologist reviewed the clinical data for all patients. For the diseasefree progression study, only patients with clinical follow-up of at least 18 months or until disease recurrence were included. The characteristics of the tumors and patient outcome for the sample sets are summarized in Table 1.

\section{Tissue microarray (TMA)}

Areas of tumor were selected based on review of a hematoxylin-eosin-stained slide. All samples were fixed with formalin and embedded in paraffin following a standard procedure. Formalin fixed paraffin embedded tumor blocks were then biopsied using a $0.6 \mathrm{~mm}$ diameter tissue arrayer and resultant cores were arrayed into a grid in a recipient paraffin block. It has previously been demonstrated using several different antibodies that the quality of the core samples on this TMA was suitable for immunohistochemistry and statistical analyses confirmed that the age of the paraffin blocks was not a confounder in these studies [18]. The tissue array was composed of 260 ovarian cancer samples from patients that never received chemotherapy before their surgery and 11 samples of areas from normal fallopian tubes of cancer patients. After review of the clinical data 68 patients were excluded from the final analysis, as they did not meet the study inclusion criteria. For the RPS4X immunostaining study, two core samples on the TMA were damaged and thus excluded (thus $\mathrm{N}=190$ ). For the YB-1 immunostaining study, six core samples were excluded for similar reason (thus $\mathrm{N}=186$ ). The completed

Table 1 Description of the high-grade serous ovarian carcinomas (HGSOC) tissue array

\begin{tabular}{ll}
\hline Variable & $\mathbf{N}=\mathbf{1 9 2}$ \\
\hline \multicolumn{1}{l}{ Stage } & $\mathrm{n}(\%)$ \\
I & $10(5.2)$ \\
III & $21(10.9)$ \\
IV & $135(70.3)$ \\
Res. Disease & $26(13.5)$ \\
Negative & \\
Milliary & $26(13.5)$ \\
$<1$ cm & $5(2.6)$ \\
1-2 cm & $29(15.1)$ \\
2 cm & $16(8.3)$ \\
Variable & $63(32.8)$ \\
& $\mathrm{N}=192$ \\
Age, years & mean (SD) \\
Disease free survival, month & $62(11)$ \\
Overall survival, month & $22(26)$ \\
\hline
\end{tabular}

Stage is divided in 4 categories according to FIGO classification. Residual disease at surgery time (Res. Disease) was evaluated by a gyneco-oncologist (in $\mathrm{cm}$ ). negative $=$ no residual disease. milliary $=$ very small, discrete, multiple areas of tumor tissue, and is grouped by convention with the $<1 \mathrm{~cm}$ group. Abbreviations: $S D$ standard deviation. 
tissue array was sectioned, stained with hematoxylin-eosin and received another pathology review to confirm tumor content [18].

\section{Immunohistochemistry}

The TMA of formalin fixed paraffin embedded tumors was sectioned at $4 \mu \mathrm{m}$ and slides were stained using the BenchMark XT automated stainer (Ventana Medical System Inc.). The optimal concentration for each primary antibody was determined by serial dilutions. The rabbit polyclonal antibody against human RPS4X (14799-1-AP) was purchased from ProteinTech Group, Inc. (Chicago IL). A polyclonal antibody against the $\mathrm{N}$-terminus portion of YB-1 (ab12148) was purchased from Abcam, Inc. (Cambridge, MA) [19,20]. The rabbit monoclonal antibody against Ki67 (RM-9106) was purchased from Lab Vision (Fremont, CA). Nuclei were counterstained with hematoxylin. Antigen retrieval was carried out with Cell Conditioning 1 (Ventana Medical System Inc.; \#950124) for $30 \mathrm{~min}$ (YB-1 and RPS4X) or $60 \mathrm{~min}$ (Ki67). Prediluted antibody was automatically dispensed, and the slides were incubated at $37^{\circ} \mathrm{C}$ for $60 \mathrm{~min}$ (YB-1 and RPS4X) or $44 \mathrm{~min}$ (Ki67). Reactions were carried out using the UltraView DAB detection kit (Ventana Medical System Inc.; \#760-091). Slides were counterstained with hematoxylin (Ventana Medical System Inc.; \#760-2021). All sections were scanned with a 20x 0.75NA objective with a resolution of $0.3225 \mu \mathrm{m}$. Substitution of the primary antibody with phosphate buffered saline served as a negative control.

\section{Staining quantification}

Tumor sections were scanned, digitally conserved, and manually visualized. For RPS4X and YB-1, a score was given to each core according to the staining intensity of the cytoplasm in the epithelial cells from 1 (weak) to 5 (strong). For both markers, no cores presented negative staining. For Ki67, cores were scored for the percentage (rounded to the nearest 5\%) of total staining. Each array was independently analyzed in a blind study by two independent observers. We use the interrating correlation (Cronbach's Alpha) to evaluate the overall correlation between the observers as described previously [18]. Inter-rating correlation was $>75 \%$ for all three proteins. The average score from the two independent observers, for each respective core, was used for analysis.

\section{YB-1 and RPS4X knock down}

The human OVCAR-3 and SK-OV-3 serous ovarian cancer cell lines were obtained from the American Type Culture Collection (ATCC). The OVCAR-3 cells were maintained in RPMI media supplemented 15\% Fetal Bovine Serum (FBS) and 1\% Penicillin-Streptomycin (Invitrogen,
Carlsbad, CA) at $37^{\circ} \mathrm{C}$ in atmosphere of $5 \% \mathrm{CO}_{2}$. The SK$\mathrm{OV}-3$ cells were maintained in DMEM supplemented with 10\% FBS and 1\% Penicillin-Streptomycin. To deplete RPS4X or YB-1 proteins in cells, small interference RNA (siRNA) molecules were transfected with the Lipofectamine 2000 as described by the manufacturer (Invitrogen, Carlsbad, CA). The knock down efficiency was confirmed by western blot analyses with antibodies against YB-1, RPS4X, and $\beta$-actin as control. Horseradish peroxidase-conjugated secondary antibodies (anti-rabbit IgG: NAV934V and anti-mouse IgG: NA931V) were purchased from GE Healthcare Limited (Piscataway, NJ). The siRNA sequences against YB-1 are 5'-AAGAA GAAAUAUGAAAUUCCA-3' for the siRNA-A molecule and 5 ' -CUGCAAGCACCUGUUAAUAAA-3' for siRNAB. The siRNA sequences against RPS4X are 5'-CAGA UCUUUGUACGUAAUUAA-3' for the siRPS4X-A molecule and 5' -CGGGAGAGAAUUUCCGUCUGA-3' for siRPS4X-D. A scrambled control siRNA was purchased from Invitrogen (Carlsbad, CA).

To obtain the growth curves of transfected cells, 10,000 OVCAR-3 or 50,000 SK-OV-3 transfected cells were plated in $60 \mathrm{~mm}$ dishes and counted with a hemacytometer by the trypan blue exclusion technique every other day. Experiments were performed in triplicate.

\section{Western blots}

All transfected and untransfected cells were lysed in RIPA buffer [50 mM Tris- $\mathrm{HCl}$ (pH 7.5), $150 \mathrm{mM} \mathrm{NaCl}$, $1 \%$ NP-40, $0.1 \%$ SDS, $0.5 \%$ sodium deoxycholate] for SDS-PAGE analyses. Proteins from SDS-PAGE were transferred onto Amersham Hybond-P membranes (GE Healthcare Limited, Piscataway, NJ). Membranes were blocked one hour at room temperature in PBS containing $5 \%$ milk $/ 0.1 \%$ Tween, washed in PBS-Tween $(0.1 \%)$, and incubated overnight with the primary antibodies in PBS containing $5 \%$ milk overnight at $4^{\circ} \mathrm{C}$. Blots were washed the next day in PBS-Tween and incubated two hours at room temperature with horseradish peroxidase-conjugated secondary antibody in PBS containing 5\% milk. Blots were washed with PBS-Tween and proteins were revealed with chemiluminescence reagents (ECL Plus; GE Healthcare Limited, Piscataway, NJ). Immunoprecipitation of GFP (Green Fluorescent Protein) and GFP-YB-1 constructs were performed as described previously [21]. Protein bands on western blots were quantified using LI-COR Image Studio software 2.0 (LI-COR Biosciences, Lincoln, NE). $\beta$-actin was used as a control for protein loading. The background signal for each band was determined using an identical area to the target band covering a region in the same lane where no protein signal was observed. Results were determined by calculating a ratio of target protein signal (minus background) over $\beta$-actin signal (minus background). 


\section{Immunofluorescence analysis}

SK-OV-3 cells were plated on coverslips and transfected the next day with control siRNA or siRNA sequences against RPS4Xm RNA. Three days later cells were fixed in $4 \%$ paraformaldehyde for $20 \mathrm{~min}$ at room temperature (RT) and permeabilized with $0.15 \%$ Triton X-100 at RT for $10 \mathrm{~min}$. After washing with PBS, cells were blocked with $3 \% \mathrm{BSA}$ at room temperature for $30 \mathrm{~min}$. After blocking, the antibody against RPS4X was diluted in $1 \%$ blocking buffer (1:100) and applied to the coverslips for an overnight incubation at $4^{\circ} \mathrm{C}$. The next day, coverslips were washed with PBS and incubated with rhodaminesecondary antibody (Santa Cruz) for $1 \mathrm{~h} 30 \mathrm{~min}$ in the dark at RT. After washing, coverslips were stained with DAPI $10 \mathrm{~min}$, washed, and mounted on glass slides. Slides were viewed at $60 \mathrm{X}$ magnification (1.4NA oil-immersion $60 \mathrm{X}$ objective) and zoomed $2 \mathrm{X}$ for image acquisition on a Nikon inverted diaphot confocal microscope equipped with Krypton/Argon lasers (488 and $568 \mathrm{~nm}$ ). Images were captured with a BioRad MRC1024 confocal microscopy system. Finally, images were analyzed (colored and merge) using the Fiji-win32 software.

\section{FACS and FITC-Annexin $V$ analyses}

Cells were transfected with either control siRNA or siRNA against RPS4X. After $72 \mathrm{~h}$, cells were fixed in 50\% ethanol overnight. Cells were then washed in phosphatebuffered saline (PBS) and incubated for $30 \mathrm{~min}$ at $37^{\circ} \mathrm{C}$ in a buffer containing propidium iodide and RNAses. Cells were then analyzed on a Beckman-Coulter Epics Elite ESP (Cambridge, MA, USA) flow activated cell sorter. Data were analyzed with the MultiCycle software (Phoenix Flow System, San Diego, CA, USA). To estimate apoptosis and/ or necrosis, we used the FITC Annexin V apoptosis detection kit I (BD Biosiences, Palo Alto, CA). Transfected cells were treated $48 \mathrm{~h}$ with the indicated concentration of cisplatin and then harvested to measure apoptosis/necrosis following the manufacturer's instructions.

\section{Cisplatin treatment and sulforhodamine B colorimetric assay}

Cells were transfected with the indicated siRNAs and allowed to grow for 24 hours. The next day, 10,000 cells were seeded per well on a 96-well plate and incubated at $37^{\circ} \mathrm{C}$ for 24 hours. Different concentrations $(0-40 \mu \mathrm{M})$ of cisplatin were added to the cells in triplicate and cells were then allowed to grow for an additional 48 hours. Cells were fixed with tricholoroacetic acid $(10 \% \mathrm{w} / \mathrm{v})$ and stained 30 min with sulforhodamine B as described [22].

\section{Statistical analysis}

The Spearman correlation (two-tailed) and non-parametric Wilcoxon-Mann-Whitney test were used to estimate the correlation with clinicopathological variables and markers as continuous variables. Survival curves were calculated according to Kaplan-Meier method coupled with a logrank test for survival analysis. Since survival times were positively skewed, we took the median as the threshold value for each marker (YB-1 and RPS4X). Univariable and multivariable Cox proportional hazard models were used to estimate the hazard ratio for each marker as continuous variables. All statistical analyses were done using Statistical Package for the Social Sciences software version 16.0 (SPSS, Inc.), and statistical significance was set at $P<0.05$.

The $\mathrm{R}$ software version 2.10.1 (http://www.r-project. $\mathrm{org} /$ ) was used to estimate the growth rate, the IC50, and the associated standard deviation. Briefly, the growth curves were fitted to a mathematical model of the form $y=x_{0} *(1+r)^{t}$, where $x_{0}$ represents the 50,000 transfected cells plated on day $0, r$ represents the growth rate, and $\mathrm{t}$ represents the time unit (days). The dose response curves were fitted to a standard exponential decay mathematical model of the form $y=y_{0}+A^{*} e^{k x}$ where $y_{0}$ represents the minimal normalized intensity, A the intensity at time 0 and $\mathrm{k}$ is the decay rate.

\section{Results}

RPS4X and YB-1 expression in ovarian cancer samples

It has been reported that YB-1 is overexpressed in ovarian cancers $[7,23]$. Since we recently found an interaction between RPS4X and YB-1 proteins [15] that may affect clinical outcomes of patients, we determined the levels of expression of these proteins by immunohistochemistry in 192 clinical samples from women with ovarian cancer (Table 1). (Note that for each immunohistochemistry staining experiments, samples of poor quality were excluded from the statistical analyses). We correlated the expression of these proteins with the mitotic index marker Ki67 from the same samples. Figure 1 demonstrates examples of the staining pattern obtained with the antibodies used against YB-1, RPS4X, and Ki67. Expression of proteins in the epithelium of the ovarian cancer tissues was observed and scored according to the intensity of staining as low to strong (1 to 5) (Figure 1). Both YB-1 and RPS4X were mainly cytoplasmic, while Ki67 gave a nuclear staining. The quality and validity of the antibodies against YB-1 and Ki67 used in this immunohistochemistry study have been described previously $[18,19]$. To confirm the validity of the antibody against RPS4X, we performed western blot and immunofluorescence tests on control and RPS4X-depleted SK-OV-3 ovarian tumor cell lines. As indicated in Figure 1D, the RPS4X antibody recognized a band of approximately $29 \mathrm{kDa}$ that was depleted by two different siRNAs specific to RPS4X mRNAs. The immunofluorescence signal was also reduced in a population of SK-OV-3 cells transfected with a siRNA against RPS4X compared to siRNA control cells (Figure 1E). These results indicate that the antibody is specific to the RPS4X protein. 


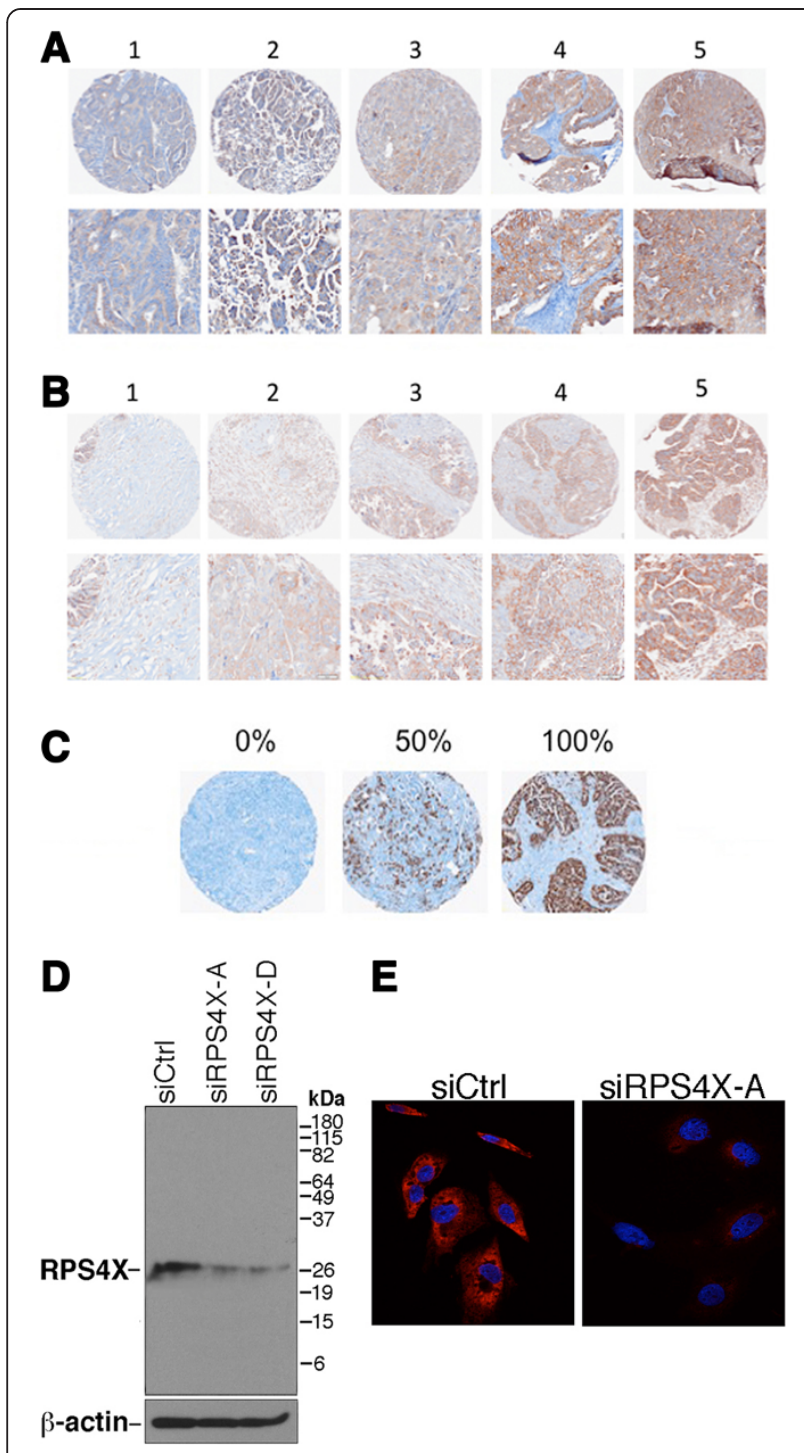

Figure 1 Representative staining for immunohistochemistry of YB-1, RPS4X, and Ki67 on a high-grade serous EOC TMA. A, representative staining of each intensity by immunohistochemistry for YB-1. From left to right: low to high intensity. B, representative staining of each intensity by immunohistochemistry for RPS4X. From left to right: low to high intensity. C, representative staining of each intensity by immunohistochemistry for Ki67. From left to right: 0\% of total staining, $50 \%$ of total staining, and $100 \%$ of total staining. D, western blot analysis of total protein extracts from SK-OV-3 cell lines transfected with a siRNA sequence against RPS4X mRNA (siRPS4X-A and siRPS4X-D) or transfected with a control (scrambled) siRNA sequence. $\beta$-actin is used as a loading control. $\mathbf{E}$, a representation of immunofluorescence signals in SK-OV-3 cells transfected with a siRNA sequence against RPS4X mRNA (siRPS4X-A) or transfected with a control scrambled siRNA sequence (siCtrl). Nuclei are revealed by DAPI staining (in blue).

We investigated whether YB-1 was associated with RPS4X and Ki67 expression in ovarian cancer. Overexpression of YB-1 correlated significantly with total expression of Ki67 and expression of RPS4X in our clinical samples (Table 2). As expected, the expression of RPS4X correlated significantly with the expression of YB-1. It also correlated positively with the expression of the mitotic index marker Ki67.

We next investigated the correlation between clinicopathological features of ovarian cancer cases and the expression of YB-1 and RPS4X. We determined whether the expression of YB-1 and RPS4X were associated with survival time and disease recurrence in patients with ovarian cancer using Kaplan-Meier plots. YB-1 was not significantly associated with either survival or recurrence time in our cohort (see Additional file 1: Figure S1). In contrast, Kaplan-Meier plots for RPS4X showed that the high expression of this biomarker is strongly associated with an increased overall patient survival $(P=0.002)$ (Figure 2A). Progression time was also significantly shorter in patients with low RPS4X expression $(P=0.0004)$ (Figure 2B). RPS4X also correlated significantly with lower levels of residual disease (Table 2) and with a lower disease stage (Table 2 and see Additional file 2: Table S2). Finally, YB-1 and RPS4X expression levels did not significantly correlate with patient age at diagnosis (Table 2).

In univariable Cox regression analysis, the level of RPS4X protein was evaluated to reflect the relation between decreasing levels of RPS4X expression and adverse prognosis. In this analysis, high expression of RPS4X is associated with a high hazard risk (HR) for survival $(\mathrm{HR}=0.713 ; 95 \%$ Confidence Interval $[\mathrm{CI}]=$ 0.583-0.873, $P=0.001$ ) (Table 3). It was also observed that higher RPS4X expression was associated with a longer time to disease progression $(\mathrm{HR}=0.761 ; 95 \% \mathrm{CI}=$ 0.652-0.888, $P=0.001)$. In multivariable Cox regression analysis, when standard prognostic variables were considered (age, stage and residual disease), RPS4X remained an independent variable predicting a high risk of survival $(\mathrm{HR}=0.689 ; 95 \% \mathrm{CI}=0.545-0.871, P=0.002)$ and a late risk of progression in the multivariable model $(\mathrm{HR}=$ 0.751; 95\%CI $=0.626-0.901, P=0.002$ ) (Table 3 ).

To summarize, all our statistical analyses indicate that high expression of RPS4X is associated with less aggressive ovarian tumors, slower disease progression, and with less deaths associated with this disease.

\section{Impact of RPS4X depletion on the growth of two serous epithelial ovarian cancer cell lines}

We examined the effect of depleting YB-1 protein on RPS4X levels in the ovarian tumor line OVCAR-3. As indicated in Figure 3, a depletion of YB-1 protein (by approximately two-fold) with two different siRNAs did not have a significant effect on RPS4X protein levels (Figure 3A and B). Two different siRNAs against our target proteins were used in all experiments to avoid confounding results 
Table 2 Spearman correlation test (two-tailed) for YB-1 and PRS4X expression (intensity) in EOC tissues and clinical data of patients

\begin{tabular}{|c|c|c|c|c|c|c|c|}
\hline & & YB-1 & RPS4X & Ki67 \% total & Stage & Age & Residual disease \\
\hline \multirow[t]{3}{*}{ YB-1 } & correlation & 1 & $0.391^{* *}$ & $0.315^{* *}$ & -0.016 & 0.004 & -0.053 \\
\hline & Sig. (2-tailed) & & $<0.001$ & $<0.001$ & 0.829 & 0.961 & 0.535 \\
\hline & $\mathrm{N}$ & 186 & 186 & 180 & 186 & 186 & 137 \\
\hline \multirow[t]{3}{*}{ RPS4X } & correlation & $0.391^{* *}$ & 1 & $0.224^{* *}$ & $-0.180^{*}$ & 0.041 & $-0.193^{*}$ \\
\hline & Sig. (2-tailed) & $<0.001$ & & 0.002 & 0.013 & 0.557 & 0.022 \\
\hline & $\mathrm{N}$ & 186 & 190 & 183 & 190 & 190 & 141 \\
\hline
\end{tabular}

* Correlation is significant at the 0.05 level (2-tailed).

** Correlation is significant at the 0.01 level (2-tailed).

Spearman correlation test were performed on clinical data, total Ki67\%, YB-1, and PRS4X staining intensity observed in the intra-epithelial area of ovarian tumors. Stage was evaluated according to the FIGO classification. "Sig." in the table represents the P-value from Spearman correlation test. N is the number of cases included in the statistical analysis.

due to potential off target effect of a single siRNA [15]. Similarly, a two-fold depletion of RPS4X protein did not have a significant effect on YB-1 protein levels (Figure 3B). These results suggest that RPS4X and YB-1 do not regulate each other at the protein expression level in OVCAR-3 cells. The depletion of RPS4X in SK-OV-3 cells with the siRPS4X-D sequence decreased YB-1 protein levels by $33 \%$

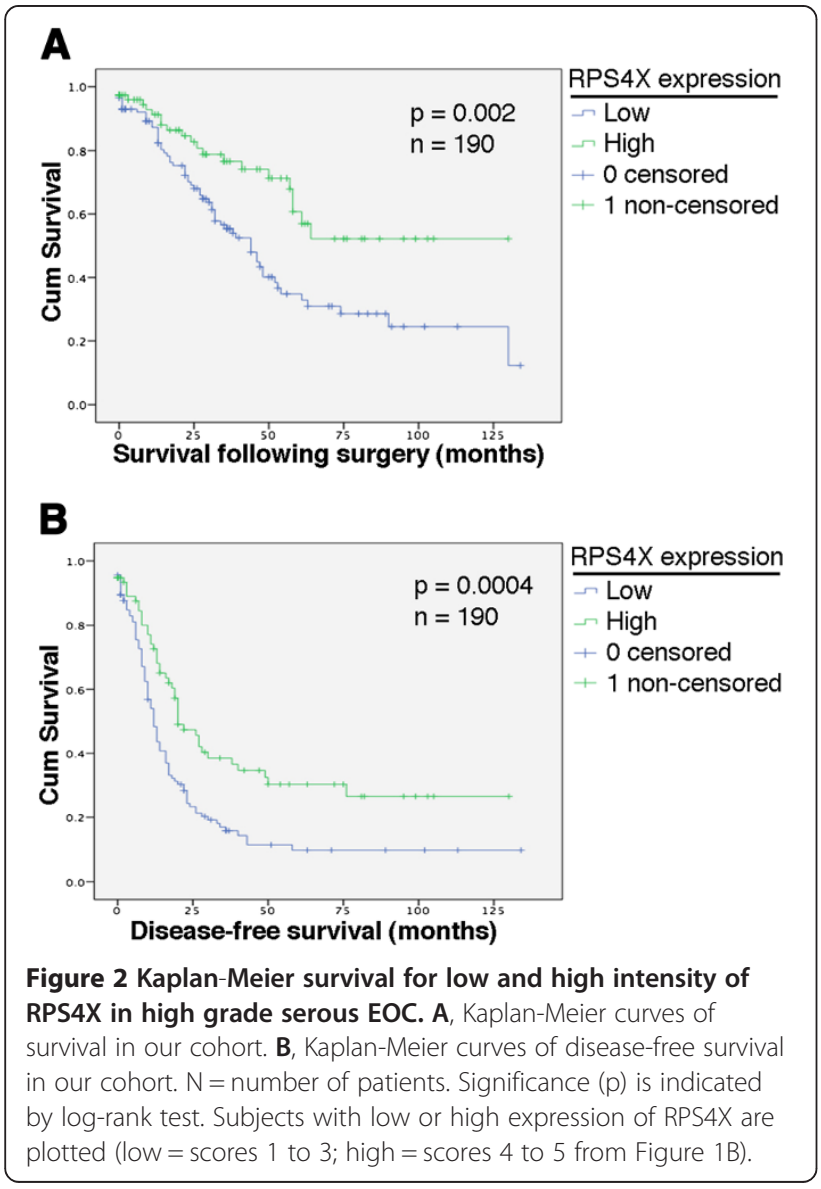

only (Figure $3 \mathrm{C}$ and $\mathrm{D}$ ). In contrast, the siRPS4X-A sequence did not decrease YB-1 protein level significantly (less than 14\%) compared to the siControl transfection based on the overlap of the error bars of the histogram in Figure 3D.

We next investigated the effect of RPS4X depletion on OVCAR-3 and SK-OV-3 cell growth. As indicated in Figure 4, two different siRNAs against RPS4X (hereafter designated siRPS4X-A and siRPS4X-D) significantly decreased the growth rate of OVCAR-3 and SK-OV-3 cells. We further analyzed the cell cycle of transfected cells by FACS analysis. As indicated in the summary histogram of Figure 4E, siRPS4X OVCAR-3 transfected with siRPS4X sequences showed an increase in $\mathrm{S}$ phase with a concomitant decrease in the G1 phase of the cell cycle compared to control siRNA transfected cells. Based on the growth rate (Figure 4C), these results suggest that the siRPS4X stalls OVCAR-3 cell proliferation in the $S$ phase of the cell cycle. SK-OV-3 transfected with siRPS4X sequences exhibited an increase in the G2/M phase of the cell cycle with a concomitant decrease in the $S$ phase. Based on the growth rate (Figure 4D), these results suggest that siRPS4X stalls SK-OV-3 cell proliferation in the G2/M phases of the cell cycle. Examples of FACS analyses are shown in the Additional file 3: Figure S2. The difference between RPS4X-depleted OVCAR-3 and SK-OV-3 cell cycle behavior is currently unknown. Nevertheless, siRPS4X decreased the proliferation rate in both cell lines.

To determine whether a depletion of RPS4X had an impact on apoptosis, we analyzed siRPS4X transfected cells with a FITC-Annexin V assay and compared them to control siRNA transfected cells. A depletion of RPS4X protein in OVCAR-3 cells did not increase the percentage of apoptotic or necrotic cells in culture (Figure 5). In contrast, RPS4X depletion in SK-OV-3 cells increased apoptosis by $17 \%$ (Figure 5 ). These results indicate that the SK-OV-3 cells are more sensitive to the depletion of RPS4X protein than the OVCAR-3 cells. 
Table 3 Cox regression analyses representing the statistical association between RPS4X expression and outcome in patients with high-grade serous ovarian cancer patients

\begin{tabular}{|c|c|c|c|c|c|c|c|}
\hline & & & ivariable anal & & & tivariable anal & \\
\hline & & HR & $(95 \% \mathrm{Cl})$ & $P$ & HR & $(95 \% \mathrm{Cl})$ & $P$ \\
\hline Survival & age & 0.998 & (0.977 1.019) & 0.841 & 0.999 & $(0.974$ 1.025) & 0.952 \\
\hline & stage & 1.713 & (1.179 2.490) & 0.005 & 1.432 & (0.804 2.552) & 0.223 \\
\hline & Res. Dis. & 1.926 & (1.490 2.491) & 0.000 & 1.732 & (1.309 2.290) & 0.000 \\
\hline & RPS4X & 0.713 & (0.583 0.873$)$ & 0.001 & 0.689 & $(0.5450 .871)$ & 0.002 \\
\hline Progression & age & 0.993 & (0.976 1.009) & 0.381 & 0.984 & (0.965 1.004) & 0.110 \\
\hline & stage & 2.238 & (1.684 2.973) & 0.000 & 1.877 & (1.257 2.805) & 0.002 \\
\hline & Res. Dis. & 1.714 & (1.425 2.060) & 0.000 & 1.552 & (1.262 1.908) & 0.000 \\
\hline & RPS4X & 0.761 & (0.652 0.888$)$ & 0.001 & 0.751 & $(0.6260 .901)$ & 0.002 \\
\hline
\end{tabular}

Res. Dis. = amount of residual disease at time of primary resection of ovarian tumor. Age $=$ age of patient at the diagnosis. $\mathrm{HR}=$ hazard ratio. $\mathrm{Cl}=\mathrm{confidence}$ interval. $P=p$ value.

Depletion of RPS4X in OVCAR-3 and SK-OV-3 cells induces cisplatin resistance

We first compared the expression of endogenous RPS4X in untransfected OVCAR-3 and SK-OV-3. As shown in Figure $6 \mathrm{~A}$ and $\mathrm{B}, \mathrm{RPS} 4 \mathrm{X}$ protein levels were 1.5 -fold higher in OVCAR-3 cells than SK-OV-3 cells. Although such cells were derived from patients with malignant ascites resistant to clinically relevant concentrations of cisplatin (www.atcc.org), we examined whether a depletion of RPS4X could increase cisplatin resistance further. As indicated in Figure 6C and D, RPS4X-depleted ovarian cancer cells were more resistant to cisplatin than control siRNA transfected cells. The calculated IC50 in OVCAR-3 cells for the control siRNA, siRPS4X-A, and siRPS4X-D were $0.9,2.7$, and $1.8 \mu \mathrm{M}$, respectively (Unpaired student $t$-test: $P=0.0084$ for siRPS4X-A $v s$ siCTRL and $P=0.025$ for siRPS4X-D vs siCTRL) (Figure 6E). The calculated IC50 in SK-OV-3 cells for the control siRNA, siRPS4X-A, and siRPS4X-D were 9.1, 25.1, and $36.3 \mu \mathrm{M}$, respectively (Unpaired student $t$-test: $P=0.00066$ for siRPS4X-A $v s$ siCTRL and $P=0.0001$ for siRPS4X-D $v s$ siCTRL) (Figure 6F). These results indicate that cells that express low levels of RPS4X are more resistant to cisplatin and a depletion of RPS4X causes further cisplatin resistance in both serous epithelial ovarian cancer cell lines tested in this study.

We next analyzed the impact of cisplatin on cell death in transfected cells with a FITC-Annexin V assay. OVCAR-3
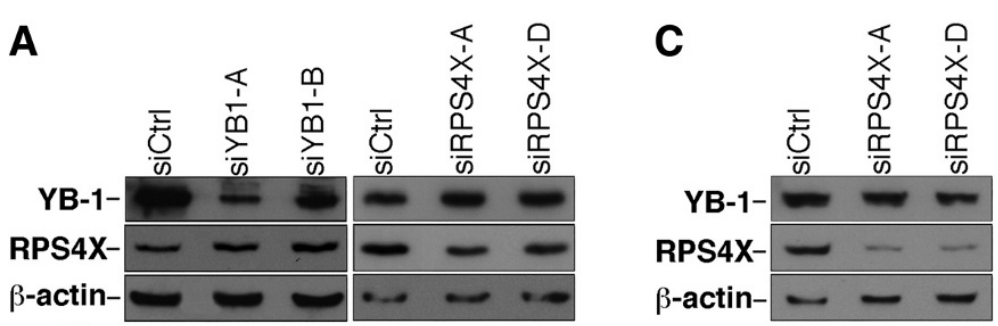

B

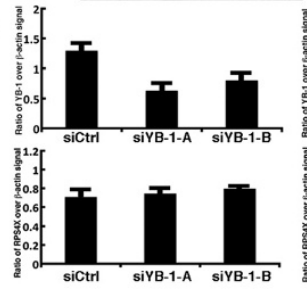

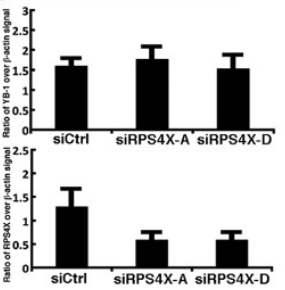

D

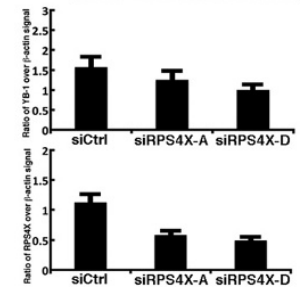

Figure 3 Western blot analysis of total protein extracts from OVCAR-3 and SK-OV-3 cell lines transfected with different siRNA sequences. A, OVCAR-3 cells were transfected with the indicated siRNA molecule and 48 hours later total protein extracts were purified, loaded on SDS/PAGE gel, and membranes were hybridized with anti-YB-1, anti-RPS4X, and anti- $\beta$-actin. Representative blots are presented. B, Histograms presenting the ratio of YB-1 or RPS4X signal over $\beta$-actin signal from the western blots in panel A. C, SK-OV-3 cells were transfected with the indicated siRNA molecule and 48 hours later total protein extracts were purified, loaded on SDS/PAGE gel, and membranes were hybridized with anti-YB-1, anti-RPS4X, and anti- $\beta$-actin. Representative blots are presented. D, Histograms presenting the ratio of YB-1 or RPS4X signal over $\beta$-actin signal from the western blots in panel C. All experiments were performed in duplicate. 


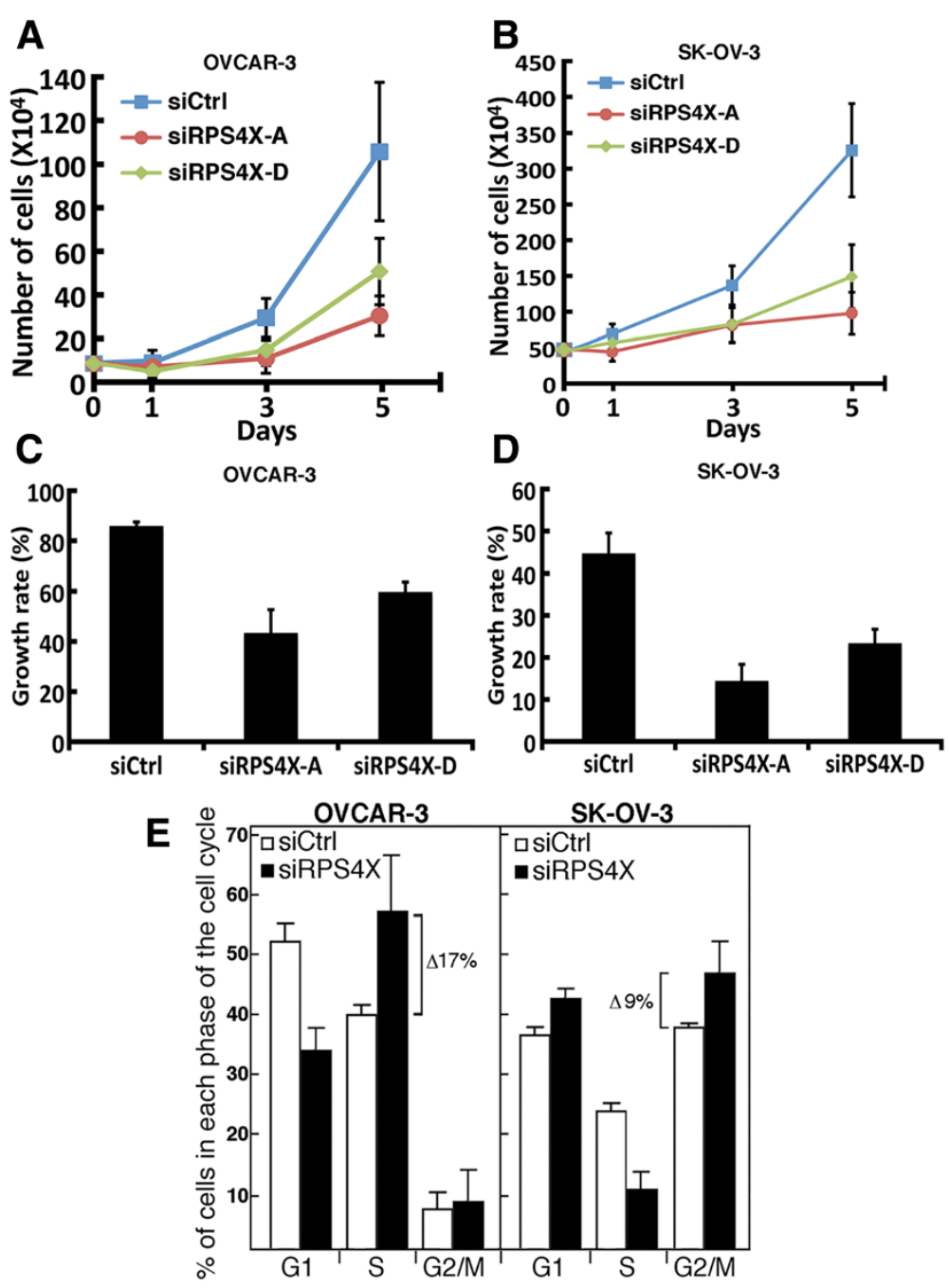

Figure 4 Cell growth in RPS4X-depleted OVCAR-3 and SK-OV-3 cells. A, cell growth of OVCAR-3 cells transfected with siCtrl, siRPS4X-A and siRPS4X-D molecules. Cells were transfected with the indicated siRNA sequences. The next day 10,000 cells were seeded in 60-mm plates and appropriate plates were counted every other day using a hemacytometer. B, Cell growth of SK-OV-3 cells transfected with siCtrl, siRPS4X-A and siRPS4X-D molecules. Cells were transfected with the indicated siRNA sequences. The next day 50,000 cells were seeded in 60-mm plates and appropriate plates counted every other day using a hemacytometer. C, Histogram representing the growth rate of OVCAR-3 transfected cells (from at least three transfections for each siRNA sequence) calculated from the growth curves in A. Error bars represent the standard deviation. (Unpaired student $t$-test: $P=7.6 \times 10^{-5}$ for siRPS4X-A vs siCTRL and $P=2.4 \times 10^{-6}$ for siRPS4X-D vs siCTRL). D, Histogram representing the growth rate of SK-OV-3 transfected cells (from at least three transfections for each siRNA sequences) calculated from the growth curves in B. Error bars represent the standard deviation (Unpaired student $t$-test: $P=3.9 \times 10^{-7}$ for siRPS4X-A vs siCTRL and $P=8.7 \times 10^{-6}$ for siRPS4X-D vs siCTRL). Growth rates were estimated as described in materials and methods. E, Percentage of OVCAR-3 and SK-OV-3 transfected cells in each phase of the cell cycle. Cells were transfected with the indicated constructs in duplicates and subjected to FACS analysis $72 \mathrm{~h}$ later. (The siRPS4X represent data from cells transfected with siRPS4X-A and siRPS4X-D performed in duplicata). Data are the mean \pm SE.

cells transfected with a control siRNA showed a $14 \%$ increase in apoptosis when treated 48 hours with $2 \mu \mathrm{M}$ cisplatin (Figure 5). There was no significant increase in necrosis. In contrast, RPS4X-depleted OVCAR-3 cells did not exhibit an increase in apoptosis or necrosis after 48 hours of cisplatin treatment. Similarly, SK-OV-3 cells transfected with a control siRNA showed a $30 \%$ and $2 \%$ increase in apoptosis and necrosis respectively when treated for 48 hours with $15 \mu \mathrm{M}$ cisplatin (Figure 5). In contrast,
RPS4X-depleted SK-OV-3 cells showed only a 7\% increase in apoptosis after 48 hours of cisplatin treatment (Figure 5). There was no increase in necrosis. Altogether these results indicate that RPS4X-depleted ovarian cancer cells are resistant to apoptosis induced by cisplatin.

\section{RPS4X interacts with YB-1 in ovarian cancer cells}

We previously showed that RPS4X interacts with a tagged YB-1 in a breast cancer cell line [15]. To confirm 

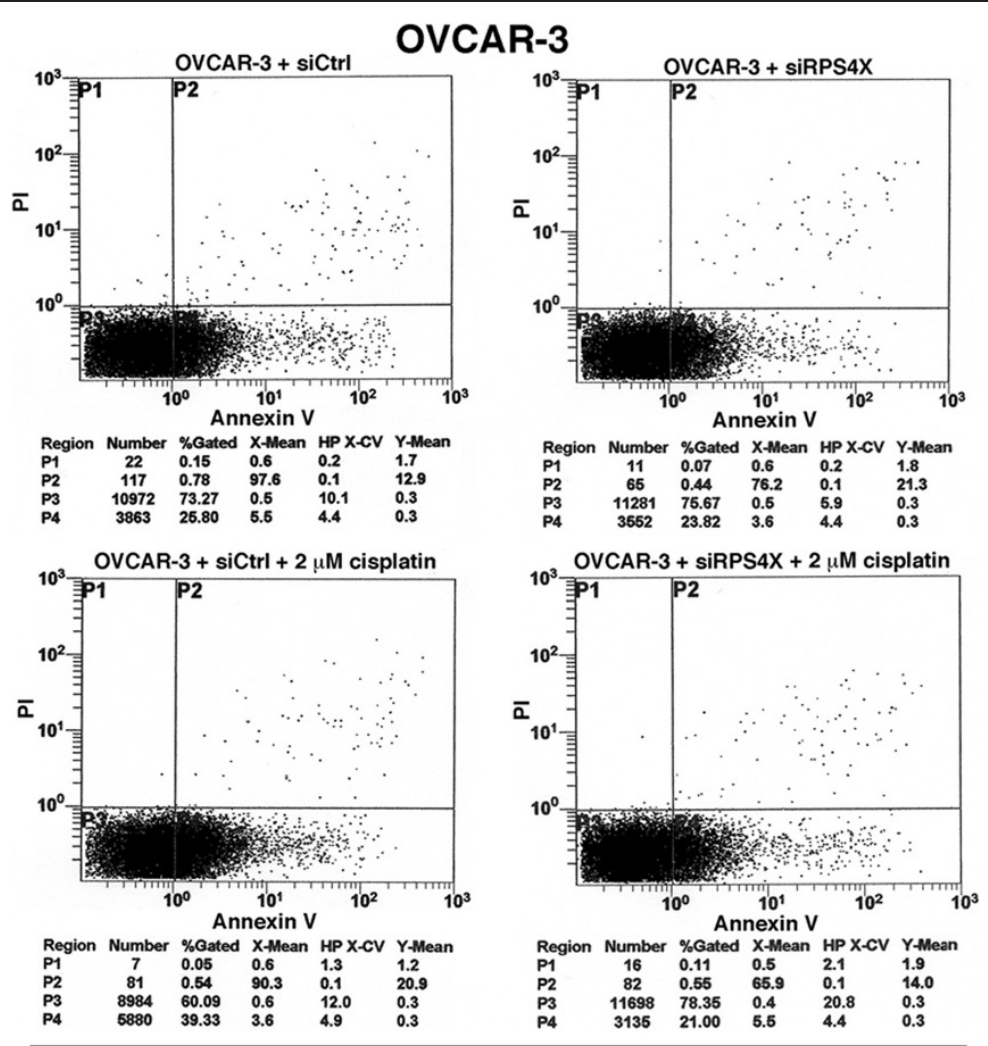

SK-OV-3
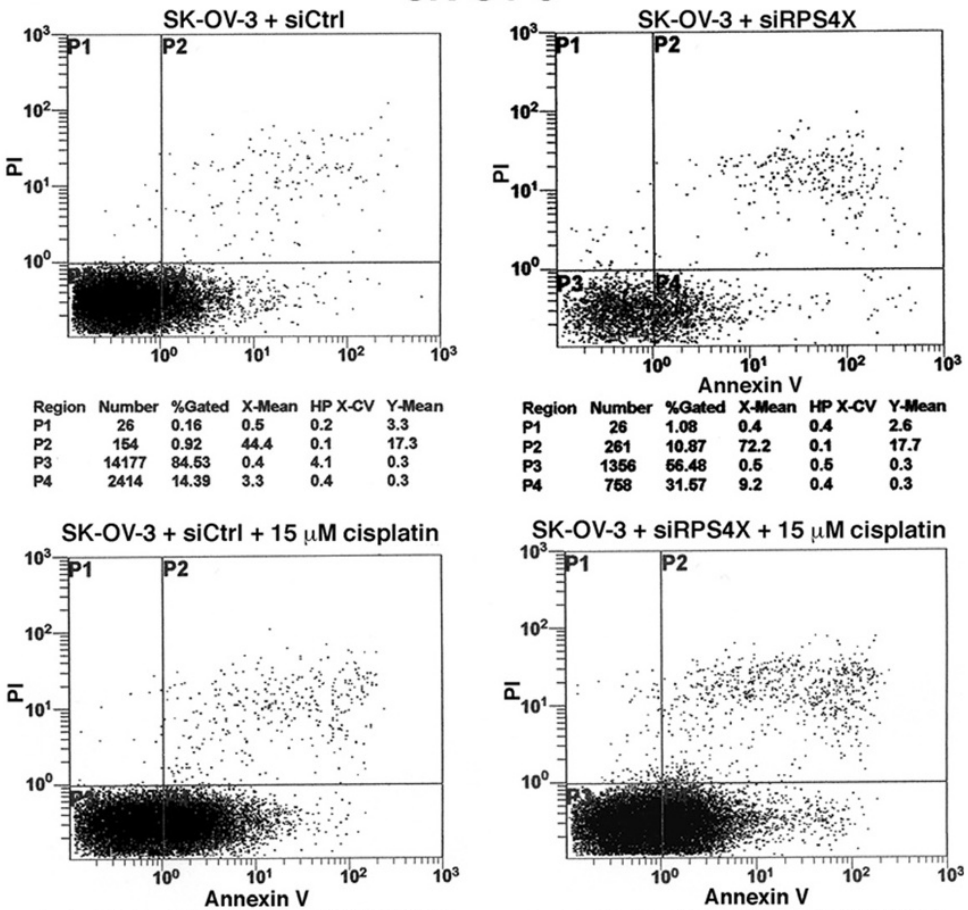

SK-OV-3 + siRPS $4 \mathrm{X}+15 \mu \mathrm{M}$ cisplatin

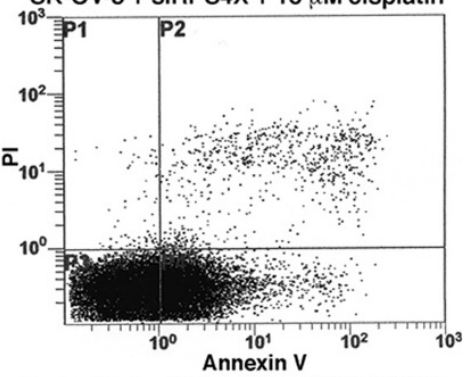

Region Number \%Gated X-Mean HPX-CV Y-Mean

$\begin{array}{llllll}\text { P1 } & 27 & 0.16 & 0.6 & 0.3 & 5.1 \\ \text { P2 } & 343 & 2.04 & 42.2 & 0.2 & 13.7 \\ \text { P3 } & 9066 & 53.84 & 0.5 & 11.6 & 0.3 \\ \text { P4 } & 7403 & 43.96 & 3.1 & 13.0 & 0.3\end{array}$

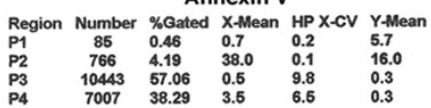

Figure $\mathbf{5}$ (See legend on next page.) 
(See figure on previous page.)

Figure 5 Apoptotic and necrotic events in RPS4X-depleted cells were assessed in the presence of cisplatin by flow cytometry with Annexin V/PI staining. Cells were transfected with the indicated siRNA sequences (siControl and siRPS4X-A) and 48 hours later cells were treated for 48 hours with the indicated concentration of cisplatin. In each graph, the P1 section (top left) represents necrotic cells, the P2 section (top right) represents both apoptotic and necrotic cells, the P3 section (bottom left) represents healthy cells, and the P4 section (bottom right) represents apoptotic cells. The number and percentage of cells in each section are indicated below each graph.

this interaction in an ovarian cancer cell line, GFP-YB-1 and a control GFP expression vectors were transfected into SK-OV-3 cells. The next day the GFP-YB-1 construct was precipitated with an antibody against the GFP tag and the presence of RPS4X in the immunoprecipitate was detected by immunoblotting (see Additional file 4: Figure S3). Endogenous RPS4X was only found in the
GFP-YB-1 immunoprecipitate indicating an interaction between RPS4X and YB-1 in ovarian cancer cells as well.

\section{Discussion}

The expression of YB-1 in ovarian carcinomas has been correlated with a poor prognosis in several studies including one focused on serous ovarian cancer $[7,23]$. In
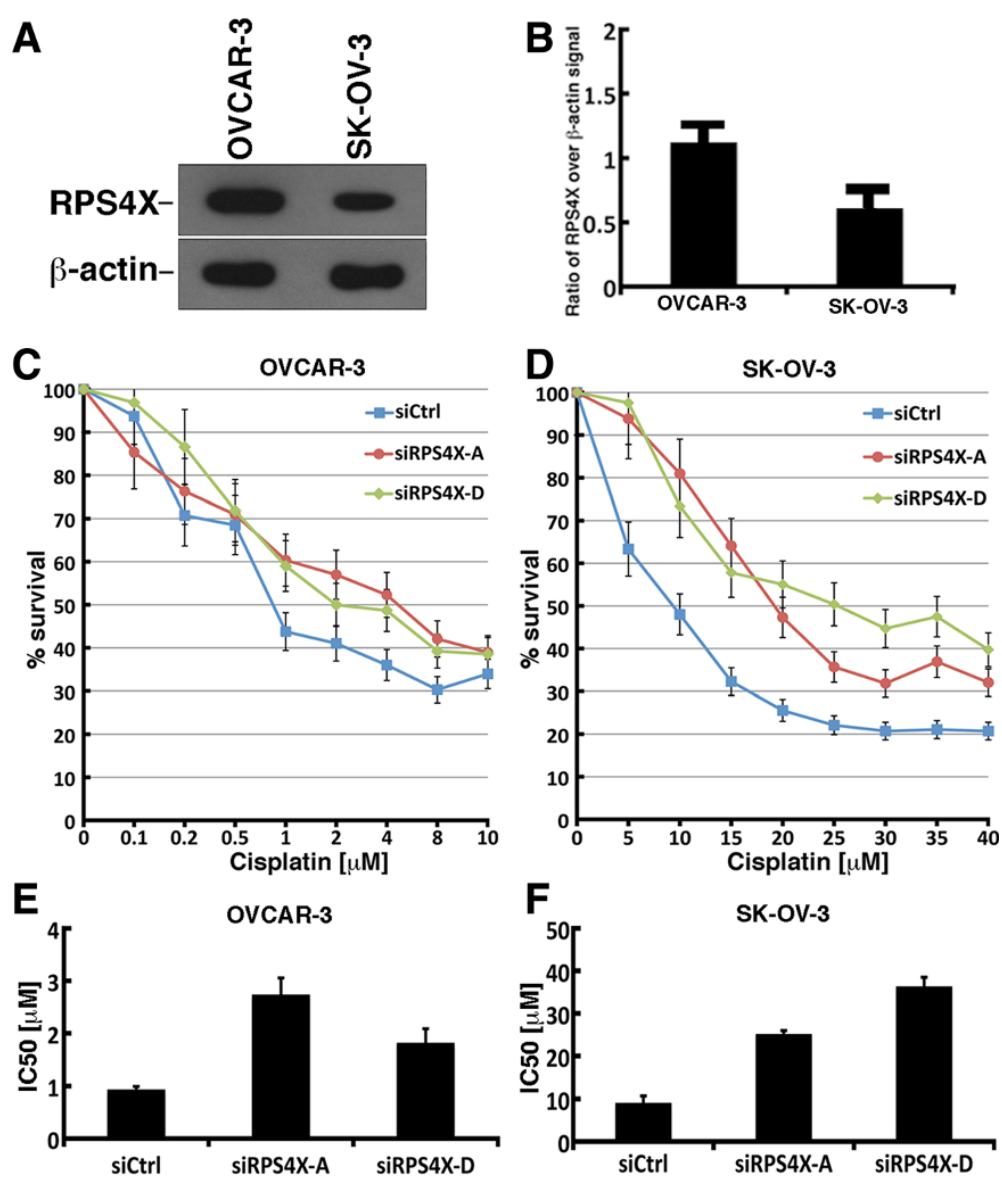

Figure 6 RPS4X depletion increases cipslatin resistance in OVCAR-3 and SK-OV-3 cells. A, One example of a western blot showing expression of RPS4X in untransfected OVCAR-3 and SK-OV-3 cells. $\beta$-actin is used as a loading control. B, Histogram presenting the ratio of RPS4X signal over $\beta$-actin signal from western blots. Experiments were performed in duplicate. C, Cisplatin dose response curves for transfected OVCAR-3 cells. D, Cisplatin dose response curves for transfected SK-OV-3 cells. Cells were transfected with the indicated siRNA molecules. Cisplatin dose response curves were determined by the sulforhodamine B colorimetric assay. E, Histogram representing the IC50 of OVCAR-3 transfected cells (from at least three transfections for each siRNA sequences) calculated from the drug response curves in $\mathrm{A}$. Error bars represent the standard deviation. (Unpaired student $t$-test: $P=0.0084$ for siRPS4X-A vs siCTRL and $P=0.025$ for siRPS4X-D vs siCTRL). F, Histogram representing the IC50 of SK-OV-3 transfected cells (from at least three transfections for each siRNA sequences) calculated from the drug response curves in B. Error bars represent the standard deviation. (Unpaired student $t$-test: $P=0.00066$ for siRPS4X-A vs siCTRL and $P=0.0001$ for siRPS4X-D vs SiCTRL). IC50 were estimated as described in materials and methods. 
contrast, there is one published report indicating no relationship between ovarian cancer patient survival and YB-1 expression [24]. Such contrasting results may be due to the small numbers of ovarian tumor samples, specifically of the serous type (less than 40 samples of both low and high grades), that were used in past studies $[7,23,24]$. Another confounding parameter in the interpretation of the results is the anti-YB-1 antibodies used in the different studies. Antibodies recognizing epitopes on the C-terminus $[7,24]$ or the $\mathrm{N}$-terminus portion of the YB-1 protein (our study) as well as the immunohistochemistry protocol can impact staining [25]. Finally, as our study focused exclusively on high-grade serous epithelial ovarian cancers, it is possible that within this subset of serous cancer YB-1 has little prognostic value. In contrast, the level of RPS4X may be a better prognostic biomarker than YB-1 in serous epithelial ovarian cancers.

Our recent analyses on YB-1 in breast cancer cell lines resistant to cisplatin have indicated an interaction between RPS4X and YB-1 [15]. As platinum-based regimen is a major treatment for ovarian cancer, we sought to determine whether the expression of RPS4X could have prognostic significance in this cancer type. In this study, we showed by immunohistochemistry that high expression of RPS4X correlated with overall survival and disease free progression. Low expression of RPS4X correlated significantly with tumor stage. These results suggest that RPS4X is a potential prognostic marker for high-grade serous epithelial ovarian cancer at the protein level. To our knowledge, there is no published study on RPS4X levels in ovarian cancers. RPS4X will need to be validated in an independent cohort of patients to confirm its clinical utility. In addition, a more quantitative way of measuring RPS4X expression, as for example real-time quantitative RT-PCR, could be envisioned.

An important aspect of RPS4X protein expression is its association with cisplatin resistance in different cell lines. The SK-OV-3 cell line is more resistant to cisplatin than the OVCAR-3 cell line [26,27]. Interestingly, the expression of endogenous RPS4X protein is lower in the more cisplatin resistant SK-OV-3 cell line than the OVCAR-3 cell line. In addition, a depletion of RPS4X in both the OVCAR-3 and SK-OV-3 ovarian cancer cell lines induced cisplatin resistance and is consistant with our previous data on RPS4X depleted breast cancer cell lines resistant to cisplatin [15]. Such results suggest that RPS4X would also have predictive values with regards to platinum-based chemotherapy. A major challenge with platinum-based regimen is that ovarian cancers can be either intrinsically resistant to treatments or will become resistant during therapy [5]. As the immunohistochemistry study was performed on serous high-grade ovarian tumors from patients who had not received chemotherapeutic treatment, the patients showing low expression of
RPS4X in their tumor tissues at surgery could correlate with an intrinsic resistance to platinum-based drugs. More precisely, cancer cells with low expression of RPS4X present in high-grade tumors that have never been in contact with platinum would correspond to cells exhibiting a pre-existing mechanism for resistance to such a drug. The exact mechanism by which a depletion of RPS4X confers cisplatin resistance is not known. One hypothesis is that depletion of RPS4X could induce a ribosomal stress which in turn leads to a slower growth rate as observed in siRPS4X transfected ovarian cancer cell lines. It has been suggested that a reduced growth rate could constitute a significant event in the survival of cancer cells following a major stress like cisplatin treatment $[28,29]$. Finally, differential translation of not only several survival factors in addition to proteins critical in the control of apoptosis during cisplatin response may be affected as well in RPS4X-depleted cells. Largescale proteomic analyses may help identifying such critical regulators in RPS4X-depleted cisplatin resistant cells. In addition, a thorough analysis of the impact of RPS4X levels on different types of reagents used in chemotherapy is also required.

\section{Conclusions}

To conclude, we have established that RPS4X is a new promising prognostic marker for patients with highgrade serous ovarian cancer. More importantly, if RPS4X is shown to be predictive of cisplatin response either alone or in combination with other markers, this could be useful when selecting first line therapies for patients with serous ovarian cancer.

\section{Additional files}

Additional file 1: Figure S1. Kaplan-Meier survival for low and high intensity of $Y B$ in high grade serous $E O C$.

Additional file 2: Table S1. Wilcoxon-Mann-Whitney test for PRS4X expression (intensity) in EOC tissues and stage and residual disease of patients.

Additional file 3: Figure S2. Examples of FACS analyses with the indicated cell lines and siRNA sequences.

Additional file 4: Figure S3. Co-imunoprecipitation of endogenous RPS4X protein with GFP-YB-1 in transfected SK-OV-3 cells. Cells were transfected with GFP or GFP-YB-1 expression vectors and the next day GFP or GFP-YB-1 proteins were immunoprecipitated with an anti-GFP antibody. Endogenous RPS4X is co-immunoprecipitated only in cells transfected with the GFP-YB-1 construct. WCE = whole cell extract; antiGFP = immunoprecipitation with an antibody against GFP. Bands corresponding to GFP-YB-1 and the endogenous YB-1 proteins are shown in the whole cell extract.

\section{Competing interests}

The authors declare that they have no competing interests.

\section{Authors' contributions}

SPT, AMMM, and ML were responsible for the conception of the study. SPT and $M L$ were responsible for the cell line studies. SPT, LM, LS, JM, ML were 
responsible for the assembly and analysis and interpretation of data. ML drafted the manuscript. LM and JM did the statistical analysis. DP provided study patients. LM and LS did the pathological assessment and interpretation of it. All authors contributed to revisions and approved the final version of the manuscript.

\section{Acknowledgements}

Clinical specimens collection was supported by the Banque de tissus et de données of the Réseau de recherche sur le cancer of the Fonds de recherche du Québec - Santé which is affiliated with the Canadian Tumour Repository Network. This work was supported in part by the Canadian Institutes of Health Research and the Cancer Research Society, Inc. to ML. SPT is a scholar of the Quebec-Clinical Research Organization in Cancer Consortium, financed by the Pfizer- FRSQ Innovation Award.

\section{Author details}

${ }^{1}$ Centre de Recherche en Cancérologie de I'Université Laval, Hôpital Hôtel-Dieu de Québec, Quebec City, QC, Canada. Institut du cancer de Montréal, Montréal, QC, Canada. ${ }^{3}$ Centre de recherche du Centre hospitalier de I'Université de Montréal (CRCHUM), Montreal, QC, Canada. ${ }^{4}$ Centre de Recherche en Cancérologie de I'Université Laval, Hôpital Hôtel-Dieu de Québec, 9 McMahon Sreet, Quebec City, QC G1R 2J6, Canada.

Received: 6 November 2012 Accepted: 20 June 2013

Published: 22 June 2013

\section{References}

1. NIH Consensus Conference: Ovarian cancer. Screening, treatment, and follow-up. NIH Consensus Development Panel on Ovarian Cancer. JAMA 1995, 273(6):491-497.

2. DiSaia PJ, Bloss JD: Treatment of ovarian cancer: new strategies. Gynecol Oncol 2003, 90(2 Pt 2):S24-S32.

3. Greenlee RT, Hill-Harmon MB, Murray T, Thun M: Cancer statistics, 2001. CA Cancer J Clin 2001, 51(1):15-36.

4. Marsh S: Pharmacogenomics of taxane/platinum therapy in ovarian cancer. Int J Gynecol Cancer 2009, 19(Suppl 2):S30-S34.

5. Kartalou M, Essigmann JM: Mechanisms of resistance to cisplatin. Mutat Res 2001, 478(1-2):23-43

6. Yahata H, Kobayashi H, Kamura T, Amada S, Hirakawa T, Kohno K, Kuwano M, Nakano H: Increased nuclear localization of transcription factor YB-1 in acquired cisplatin-resistant ovarian cancer. J Cancer Res Clin Oncol 2002, 128(11):621-626.

7. Oda Y, Ohishi Y, Basaki Y, Kobayashi H, Hirakawa T, Wake N, Ono M, Nishio K, Kuwano M, Tsuneyoshi M: Prognostic implications of the nuclear localization of Y-box-binding protein-1 and CXCR4 expression in ovarian cancer: their correlation with activated Akt, LRP/MVP and P-glycoprotein expression. Cancer Sci 2007, 98(7):1020-1026.

8. Swamynathan SK, Nambiar A, Guntaka RV: Role of single-stranded DNA regions and Y-box proteins in transcriptional regulation of viral and cellular genes. FASEB J 1998, 12(7):515-522.

9. Stickeler E, Fraser SD, Honig A, Chen AL, Berget SM, Cooper TA: The RNA binding protein $\mathrm{YB}-1$ binds $\mathrm{A} / \mathrm{C}$-rich exon enhancers and stimulates splicing of the CD44 alternative exon v4. EMBO J 2001, 20(14):3821-3830

10. Ashizuka M, Fukuda T, Nakamura T, Shirasuna K, Iwai K, Izumi H, Kohno K, Kuwano M, Uchiumi T: Novel translational control through an ironresponsive element by interaction of multifunctional protein YB-1 and IRP2. Mol Cell Biol 2002, 22(18):6375-6383.

11. Evdokimova V, Ruzanov P, Anglesio MS, Sorokin AV, Ovchinnikov LP, Buckley J, Triche TJ, Sonenberg N, Sorensen PH: Akt-mediated YB-1 phosphorylation activates translation of silent mRNA species. Mol Cell Biol 2006, 26(1):277-292.

12. Ise T, Nagatani G, Imamura T, Kato K, Takano H, Nomoto M, Izumi H, Ohmori H, Okamoto T, Ohga T, et al: Transcription factor Y-box binding protein 1 binds preferentially to cisplatin-modified DNA and interacts with proliferating cell nuclear antigen. Cancer Res 1999, 59(2):342-346.

13. Pestryakov P, Zharkov DO, Grin I, Fomina EE, Kim ER, Hamon L, Eliseeva IA, Petruseva IO, Curmi PA, Ovchinnikov LP, et al: Effect of the multifunctional proteins RPA, YB-1, and XPC repair factor on AP site cleavage by DNA glycosylase NEIL1. J Mol Recognit 2012, 25(4):224-233.

14. Guay D, Garand C, Reddy S, Schmutte C, Lebel M: The human endonuclease III enzyme is a relevant target to potentiate cisplatin cytotoxicity in Y-box-binding protein-1 overexpressing tumor cells. Cancer Sci 2008, 99(4):762-769.

15. Garand C, Guay D, Sereduk C, Chow D, Tsofack SP, Langlois M, Perreault E, Yin $\mathrm{HH}$, Lebel $\mathrm{M}$ : An integrative approach to identify YB-1-interacting proteins required for cisplatin resistance in MCF7 and MDA-MB-231 breast cancer cells. Cancer Sci 2011, 102(7):1410-1417.

16. Heintz AP, Odicino F, Maisonneuve P, Quinn MA, Benedet JL, Creasman WT, Ngan HY, Pecorelli S, Beller U: Carcinoma of the ovary. FIGO 26th Annual Report on the Results of Treatment in Gynecological Cancer. Int $J$ Gynaecol Obstet 2006, 95(Suppl 1):S161-S192.

17. Rustin GJ, Vergote I, Eisenhauer E, Pujade-Lauraine E, Quinn M, Thigpen T, du Bois A, Kristensen G, Jakobsen A, Sagae S, et al: Definitions for response and progression in ovarian cancer clinical trials incorporating RECIST 1.1 and CA 125 agreed by the Gynecological Cancer Intergroup (GCIG). Int J Gynecol Cancer 2011, 21(2):419-423.

18. Le Page C, Ouellet V, Quinn MC, Tonin PN, Provencher DM, Mes-Masson AM: BTF4/BTNA3.2 and GCS as candidate mRNA prognostic markers in epithelial ovarian cancer. Cancer Epidem Biomar 2008, 17(4):913-920.

19. Chatterjee M, Rancso C, Stuhmer T, Eckstein N, Andrulis M, Gerecke C, Lorentz H, Royer HD, Bargou RC: The Y-box binding protein YB-1 is associated with progressive disease and mediates survival and drug resistance in multiple myeloma. Blood 2008, 111(7):3714-3722.

20. Otsuka Y, Kedersha NL, Schoenberg DR: Identification of a cytoplasmic complex that adds a cap onto 5'-monophosphate RNA. Mol Cell Biol 2009, 29(8):2155-2167.

21. Guay D, Gaudreault I, Massip L, Lebel M: Formation of a nuclear complex containing the p53 tumor suppressor, YB-1, and the Werner syndrome gene product in cells treated with UV light. Int J Biochem Cell Biol 2006, 38(8):1300-1313.

22. Vichai V, Kirtikara K: Sulforhodamine B colorimetric assay for cytotoxicity screening. Nat Protoc 2006, 1(3):1112-1116.

23. Kamura T, Yahata H, Amada S, Ogawa S, Sonoda T, Kobayashi H, Mitsumoto M, Kohno K, Kuwano M, Nakano $\mathrm{H}$ : Is nuclear expression of $\mathrm{Y}$ box-binding protein-1 a new prognostic factor in ovarian serous adenocarcinoma? Cancer 1999, 85(11):2450-2454.

24. Huang X, Ushijima K, Komai K, Takemoto Y, Motoshima S, Kamura T, Kohno K: Co-expression of $Y$ box-binding protein-1 and P-glycoprotein as a prognostic marker for survival in epithelial ovarian cancer. Gynecol Oncol 2004, 93(2):287-291.

25. Woolley AG, Algie M, Samuel W, Harfoot R, Wiles A, Hung NA, Tan PH, Hains P, Valova VA, Huschtscha L, et al: Prognostic association of YB-1 expression in breast cancers: a matter of antibody. PLoS One 2011, 6(6):e20603.

26. Gibb RK, Taylor DD, Wan T, O'Connor DM, Doering DL, Gercel-Taylor C: Apoptosis as a measure of chemosensitivity to cisplatin and taxol therapy in ovarian cancer cell lines. Gynecol Oncol 1997, 65(1):13-22.

27. Egawa-Takata T, Endo H, Fujita M, Ueda Y, Miyatake T, Okuyama H, Yoshino K, Kamiura S, Enomoto T, Kimura T, et al: Early reduction of glucose uptake after cisplatin treatment is a marker of cisplatin sensitivity in ovarian cancer. Cancer Sci 2010, 101(10):2171-2178.

28. LaRue KE, Khalil M, Freyer JP: Microenvironmental regulation of proliferation in multicellular spheroids is mediated through differential expression of cyclindependent kinase inhibitors. Cancer Res 2004, 64(5):1621-1631.

29. Xing H, Wang S, Hu K, Tao W, Li J, Gao Q, Yang X, Weng D, Lu Y, Ma D: Effect of the cyclin-dependent kinases inhibitor p27 on resistance of ovarian cancer multicellular spheroids to anticancer chemotherapy. J Cancer Res Clin Oncol 2005, 131(8):511-519.

\section{doi:10.1186/1471-2407-13-303}

Cite this article as: Tsofack et al.: Low expression of the X-linked ribosomal protein S4 in human serous epithelial ovarian cancer is associated with a poor prognosis. BMC Cancer 2013 13:303. 